\title{
Renormalized frequency shift of a Wannier exciton in a one-dimensional system
}

\author{
Yueh-Nan Cher $*$ and Der-San Chuv \\ Department of Electrophysics, National Chiao-Tung University, Hsinchu 300, Taiwan
}

(Dated: November 5, 2018)

\begin{abstract}
The radiative frequency shift of superradiant exciton in a one-dimensional system is calculated. It is shown that a finite frequency shift can be obtained after proper renormalization. The value of the shift is inversely proportional to the factor $\frac{\lambda}{d}$, where $\lambda$ is the wavelength of the emitted photon and $d$ is the lattice spacing.

PACS: 42.50.Fx, 32.70.Jz, 71.35.-y, 71.45.-d
\end{abstract}

Since Dicke proposed the phenomenon of superradiance 1], the coherent effect for spontaneous radiation of various systems has attracted extensive interest both theoretically and experimentally 2, 3]. In semiconductor systems, the electron-hole pair is naturally a candidate for examining the spontaneous emission. However, as it was well known, the excitons in a three dimensional system will couple with photons to form polaritons-the eigenstate of the combined system consisting of the crystal and the radiation field which does not decay radiatively [4]. Thus, in a bulk crystal, the exciton can only decay via impurity, phonon scatterings, or boundary effects.

The exciton can render radiative decay in lower dimensional systems such as quantum wells, quantum wires, or quantum dots as a result of broken symmetry. In 1966, V. M. Agranovich et al. predicted that the decay rate of the exciton is superradiantly enhanced by a factor of $(\lambda / d)^{2}$ for a 2D exciton-polariton system [5], where $\lambda$ is the wave length of the emitted photon and $d$ is the lattice constant of the thin film. First observation of superradiant short lifetimes of excitons was performed by $\mathrm{Y}$. Aaviksoo et al. on surface states of the anthracene [6]. In the past decades, the superradiance of excitons in these quantum well structures has been investigated intensively 7, 8, 9, 10, 11. For lower dimensional systems, the decay rate of the exciton is enhanced by a factor of $\lambda / d$ in a quantum wire [5]. In the quantum dots, the decay rate is shown to be proportional to $\mathrm{R}^{2.1}[12$ which confirms the theoretical prediction 13,14$]$. In fact, superradiance is accompanied by frequency shift, as pointed out in Ref. [15]. Although the spectrum of polaritons in one-dimensional state was studied in Refs. 5, 16], the radiative correction to the frequency of the spontaneous radiation from the Wannier exciton in a one-dimensional system has not been displayed explicitly. The reason is that the radiation correction to the frequency shift usually contains divergences which have to be removed by renormalization. In this paper, we show that the radiative frequency shift of the Wannier exciton can be properly renormalized in a one-dimensional system. The renormalized frequency shift of the Wannier

\footnotetext{
*Electronic address: ynchen.ep87g@nctu.edu.tw
}

$\dagger$ Electronic address: dschuu@cc.nctu.edu.tw exciton in 1-D is also superradiatively enhanced due to the coherent effect.

Consider now a Wannier exciton in a one-dimensional system with lattice spacing $d$. We will assume a twoband model for the band structure of the system. The state of the Wannier exciton with coherent length $L_{c}$ can be phenomenologically approximated as

$$
\left|k_{z}, n\right\rangle=\sum_{l \rho} \sqrt{\frac{d}{L_{c}}} \exp \left(i k_{z} r_{c}\right) F_{n}(l)
$$

where $k_{z}$ is the crystal momentum on the chain direction characterizing the motion of the exciton, $n$ is the quantum number for the internal structure of the exciton, and, in the effective mass approximation, $r_{c}=\frac{m_{e}^{*}(l+\rho)+m_{h}^{*} \rho}{m_{e}^{*}+m_{h}^{*}}$ is the center of mass of the exciton. $F_{n}(l)$ is the hydrogenic wave function with $l+\rho$ and $\rho$ being the positions of the electron and hole, respectively. Here $m_{e}^{*}$ and $m_{h}^{*}$ are, respectively, the effective masses of the electron and hole. If one neglects the effects of imperfections and scatterings, the coherent length in Eq. (1) should become infinite.

The interaction between the exciton and the photon can be written in the form

$$
H^{\prime}=\sum_{k_{z} n} \sum_{\mathbf{q}^{\prime} k_{z}^{\prime}} D_{\mathbf{q}^{\prime} k_{z}^{\prime} k_{z} n} b_{k_{z}^{\prime} \mathbf{q}^{\prime}} c_{k_{z} n}^{\dagger}+\text { H.c. }
$$

where

$$
D_{\mathbf{q}^{\prime} k_{z}^{\prime} k_{z} n}=\frac{e}{m c} \sqrt{\frac{2 \pi \hbar c L_{c}}{\left(q^{\prime 2}+k_{z}^{\prime 2}\right)^{1 / 2} d v}} \epsilon_{\mathbf{q}^{\prime} k_{z}^{\prime}} \chi_{k_{z} n}
$$

with $\epsilon_{\mathbf{q}^{\prime} k_{z}}$ being the polarization of the photon. $c_{k_{z} n}$ and $b_{\mathbf{q}^{\prime} k_{z}^{\prime}}$ are the operators of the exciton and photon, respectively. $E_{k_{z} n}$ is the exciton dispersion. In Eq. (3),

$$
\begin{aligned}
\chi_{k_{z} n}= & \sum_{l} F^{*}(l) \int d \tau \omega_{c}(\tau-l) \exp \left(i k_{z}\left(\tau-\frac{m_{e}^{*}}{m_{e}^{*}+m_{h}^{*}}\right) l\right) \\
& \times\left(-i \hbar \frac{\partial}{\partial \tau}\right) \omega_{v}(\tau)
\end{aligned}
$$

is the effective transition dipole matrix element between the electronic Wannier state $\omega_{c}$ in the conduction band and the Wannier hole state $\omega_{v}$ in the valence band. 
Because of the presence of exciton-photon interaction, the radiative decay of the exciton is expected to keep dual merits of coherent nature in the wire direction and superradiant decay perpendicular to the wire. In the interaction picture, the state $|\psi(t)\rangle$ for the whole system composed of the exciton (with frequency shift $\Omega_{k_{z} n}$ and finite decay rate $\gamma_{k_{z} n}$ ) and photons can be written as

$|\psi(t)\rangle=e^{-i \Omega_{k_{z} n} t-\frac{1}{2} \gamma_{k_{z} n} t}\left|k_{z}, n ; 0\right\rangle+\sum_{\mathbf{q}^{\prime} k_{z}^{\prime}} f_{G ; \mathbf{q}^{\prime} k_{z}^{\prime}}(t)\left|G ; \mathbf{q}^{\prime} k_{z}^{\prime}\right\rangle$

where $\left|k_{z}, n ; 0\right\rangle$ is the state with a Wannier exciton in the mode $k_{z}, n$ in the linear chain without photons, and $\left|G ; \mathbf{q}^{\prime} k_{z}^{\prime}\right\rangle$ represents the state in which the electron-hole pair recombines and a photon in the mode $\mathbf{q}^{\prime}, k_{z}^{\prime}$ is created.

The decay rate and frequency shift can be evaluated as 17

$$
\gamma_{k_{z} n}=2 \pi \sum_{\mathbf{q}^{\prime} k_{z}^{\prime}}\left|D_{\mathbf{q}^{\prime} k_{z}^{\prime} k_{z} n}\right|^{2} \delta\left(\omega_{\mathbf{q}^{\prime} k_{z}^{\prime} k_{z} n}\right)
$$

and

$$
\Omega_{k_{z} n}=\mathcal{P} \sum_{\mathbf{q}^{\prime} k_{z}^{\prime}} \frac{\left|D_{\mathbf{q}^{\prime} k_{z}^{\prime} k_{z} n}\right|^{2}}{\omega_{\mathbf{q}^{\prime} k_{z}^{\prime} k_{z} n}}
$$

where $\omega_{\mathbf{q}^{\prime} k_{z}^{\prime} k_{z} n}=E_{k_{z} n} / \hbar-c \sqrt{q^{\prime 2}+k_{z}^{\prime 2}}$ and $\mathcal{P}$ means the principal value of the integral. Thus, the Wannier exciton decay rate in the optical region can be calculated straightforwardly and is given by

$$
\begin{aligned}
\gamma_{k_{z} n}= & \frac{3 d}{4 k_{0}} \gamma_{0} \int d q^{\prime} \frac{\left|\boldsymbol{\epsilon}_{\mathbf{q}^{\prime} k_{z}^{\prime}} \chi_{n}\right|^{2}}{\left|\chi_{n}\right|^{2}} \frac{q^{\prime}}{L_{c} \sqrt{k_{0}^{2}-q^{\prime 2}}} \\
& \times \frac{\sin ^{2}\left(L_{c} \sqrt{k_{0}^{2}-q^{\prime 2}} / 2\right)}{\sin ^{2}\left(d \sqrt{k_{0}^{2}-q^{\prime 2}} / 2\right)},
\end{aligned}
$$

where $k_{0}=E_{k_{z} n} / \hbar=2 \pi / \lambda$,

$$
\chi_{n}=\sum_{l} F_{n}^{*}(l) \int d \tau w_{c}(\tau-l)\left(-i \hbar \frac{\partial}{\partial \tau}\right) w_{v}(\tau)
$$

and

$$
\gamma_{0}=\frac{4 e^{2} \hbar k_{0}}{3 m^{2} c^{2}}\left|\chi_{n}\right|^{2}
$$

with $\gamma_{0}$ being the decay rate of an isolated atom. Fig. 1 shows the numerical calculations of Eq. (8). As can be seen in the figure, the decay rate of the exciton is increased with the increase of the lateral size of the exciton center-of-mass wave function, called the exciton coherent length, and saturates when the coherence length reaches the wavelength $\lambda$ of the emitted photon. This is because as $L_{c}>\lambda$, the superradiant effect becomes prominent, and the decay rate should approach 1D limit: $\gamma_{1 d}=\frac{3 \lambda}{2 d} \gamma_{0} \frac{\left|\epsilon_{\mathbf{q}^{\prime} k_{z}^{\prime}} \chi_{n}\right|^{2}}{\left|\chi_{n}\right|^{2}} \cdot[5,17]$ Generally speaking, the enhanced factor $\lambda / d$ for perfect $1 \mathrm{D}$ crystal and $(\lambda / d)^{2}$ for $2 \mathrm{D}$ film has a simple physical meaning-it is determined by the number of unit cells in the so-called cooperative length $\lambda$.

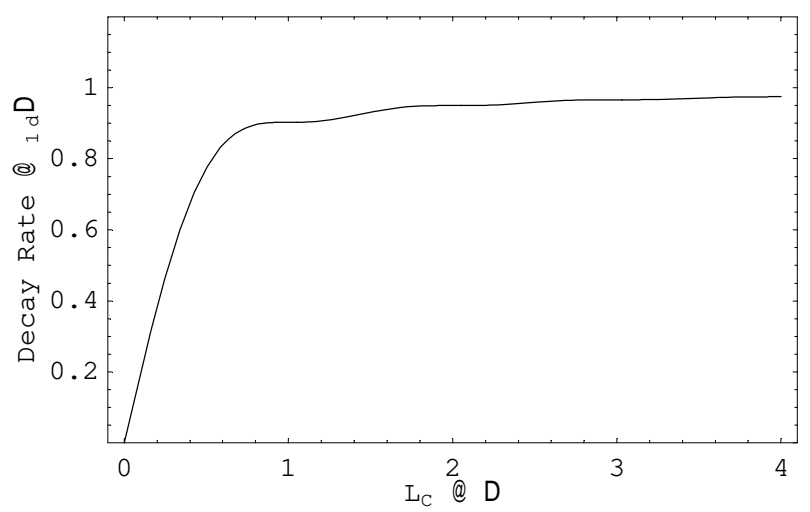

FIG. 1: Decay rate of an exciton as a function of coherent length $L_{c}$. The vertical and horizontal units are $\frac{3 \lambda}{2 d} \gamma_{0} \frac{\left|\epsilon_{\mathbf{q}^{\prime} k_{z}^{\prime}} \chi_{n}\right|^{2}}{\left|\chi_{n}\right|^{2}}$ and $\lambda$, respectively. In this graph we have assumed $\lambda=8000 \stackrel{\circ}{\AA}$ and lattice spacing $d=5 \stackrel{\circ}{A}$.

Now let us present our results for the renormalized frequency shift. For simplicity, let us consider the case that the coherent length $L_{c}$ is much larger than the wavelength of the emitted photon. Thus, the frequency shift in Eq. (7) can be expressed as

$$
\Omega_{k_{z} n}=\frac{2 \pi e^{2} \hbar L_{c}}{m^{2} c^{2} d v} \mathcal{P} \sum_{\mathbf{q}^{\prime}} \frac{\left|\epsilon_{\mathbf{q}^{\prime} k_{z}} \chi_{n}\right|^{2}}{\left(k_{0}-\sqrt{q^{\prime 2}+k_{z}^{2}}\right) \cdot \sqrt{q^{\prime 2}+k_{z}^{2}}}
$$

As can be seen from the above expression, the frequency shift suffers from infrared divergence when the denominator approaches zero. If one uses the usual procedure of mass renormalization in atomic physics, it seems the divergence becomes more strongly. To resolve this question, we adopt the method of renormalization for a system of two-level atoms proposed by Lee and Lin[18]. In their works, they identified that the frequency shift is the radiative level shift and is given by

$\Delta E_{0}=\mathcal{P} \sum_{\mathbf{q}^{\prime}} \frac{\left\langle k_{z}, n ; 0\left|H^{\prime}\right| G ; \mathbf{q}^{\prime} k_{z}\right\rangle\left\langle G ; \mathbf{q}^{\prime} k_{z}\left|H^{\prime}\right| k_{z}, n ; 0\right\rangle}{\hbar c\left(k_{0}-\sqrt{q^{2}+k_{z}^{2}}\right)}$,

where $H^{\prime}$ is defined in Eq. (2). On the other hand, they also recognized the true radiative correction to the energy 
level $\alpha$ is

$$
\Delta E=\Delta E_{0}+\left\langle\alpha\left|\theta_{c}\right| \alpha\right\rangle
$$

where $\Delta E_{0}$ is the unrenormalized level shifts, and $\theta_{c}$ is the counter-term operator. Assuming the recoil of the electron upon emitting or absorbing a photon to be negligible, one can make the following identification of the operator

$$
\theta_{c}=H^{\prime} \frac{1}{H_{p h}} H^{\prime}
$$

Therefore, the renormalized result $\left(k_{z} \sim 0\right)$ can be obtained as

$$
\Omega_{k_{z} \sim 0, n}^{r e n}=\frac{e^{2} \hbar k_{0}}{m^{2} c^{2} d} \mathcal{P} \int_{0}^{k_{m}} \frac{\left|\epsilon_{\mathbf{q}^{\prime} k_{z}} \chi_{n}\right|^{2}}{\left(k_{0}-q^{\prime}\right) q^{\prime}} d q^{\prime},
$$

where the upper limit of integration is cut off at $k_{m}$, which is taken to be the inverse of the electron Compton wavelength as usually done in the nonrelativistic cases. As one can note from Eq. (15), there is divergent problem when $q^{\prime} \sim 0$ or $q^{\prime} \sim k_{0}$. This can be overcome by substituting $-i \hbar \frac{\partial}{\partial \tau}$ by $-i m c q^{\prime} \tau$ (Ref. [15]) in Eq. (9) when $q^{\prime}$ is small. It is equivalent to the dipole-interaction form, $H^{\prime} \sim \mathbf{r} \cdot \mathbf{E}$. With this treatment, we have

$$
\Omega_{k_{z} \sim 0, n}^{r e n}=\mathcal{P} \int_{0}^{k_{m}} B_{q^{\prime} k_{z} n} d q^{\prime}
$$

with

$$
B_{q^{\prime} k_{z} n}=\left\{\begin{array}{l}
\frac{e^{2} \hbar k_{0}}{m^{2} c^{2} d q^{\prime}}\left|\epsilon_{\mathbf{q}^{\prime} k_{z}} \chi_{n}\right|^{2}, \text { when } q^{\prime} \text { is large } \\
\frac{e^{2} \hbar k_{0} q^{\prime}}{d}\left|\epsilon_{\mathbf{q}^{\prime} k_{z}} \kappa_{n}\right|^{2}, \text { when } q^{\prime} \text { is small }
\end{array}\right.
$$

where

$$
\kappa_{n}=\sum_{l} F_{n}^{*}(l) \int d \tau w_{c}(\tau-l)(-i \tau) w_{v}(\tau)
$$

In general, the wavelength of the photon is much larger than the Compton wavelength of the electron. Therefore, the renormalized frequency shift can be approximated as

$$
\Omega_{k_{z} \sim 0, n}^{r e n}=-\gamma_{\text {sin } g l e}\left(\frac{1}{k_{0} d}\right)
$$

where

$$
\gamma_{\text {sin } g l e}=\frac{2 e^{2} E_{k_{z} \sim 0, n}}{c}\left|k_{0} \kappa_{n}\right|^{2}
$$

is roughly equal to the radiative decay rate of a single isolated atom. As can be seen from Eq. (19), the renormalized frequency shift is enhanced by the factor of $\left(\frac{1}{k_{0} d}\right)$ in a single quantum wire, while the frequency shift of the exciton in a thin semiconductor film is inversely proportional to the square of the factor $k_{0} d$ [15]. We then conclude that in low dimensional systems the renormalized frequency shift of the superradiant exciton is enhanced by the factor of $\left(\frac{1}{k_{0} d}\right)^{x}$, where $x$ is the dimension of the system.

A few remarks about the differences between the previous and our works can be mentioned here. Since we consider the spontaneous emission of the exciton in the resonance approximation, the non-resonant interaction between the exciton and free photons has been omitted. Under this condition, one immediately meets the divergent problems both in 2D and $1 \mathrm{D}$ cases after performing first order perturbation. In a recent paper by V. V. Popov et al. [20], the radiative decay of coherent polariton modes in a two-dimensional excitonic system is analyzed. The authors state their approach is suitable for the examinations of time-resolved spontaneous emission. In fact, this is also the case by using our approach, where the renormalized procedure is borrowed from atomic physics. For 2D systems and in the case of normal emission $\left(k_{\|}=0\right)$, the finite (renormalized) frequency shift from our approach is also superradiant enhanced and can be written as

$$
f_{2 D} \approx-\gamma_{0}\left(\frac{\lambda_{0}}{2 \pi d}\right)^{2} L_{z}
$$

where $L_{z}$ is the well width, $\lambda_{0}$ is the wavelength of the emitted photon, $d$ is lattice spacing, and $\gamma_{0}$ is radiative decay rate of a single isolated exciton. This value agrees well with the V. V. Popov's calculation in L and $\mathrm{T}$ modes 21$]$

$$
\omega-\omega_{0}-i \gamma_{\Gamma}=-\frac{i}{2} k_{z} L_{z} \omega_{L T}
$$

where $\omega_{L T}$ means the (superradiant-enhanced) coupling strength. Since $k_{z}$ is a complex-valued quantity, hence the value $L_{z} \omega_{L T}\left(\operatorname{Im} k_{z}\right) / 2$ corresponds to our renormalized frequency shift $f_{2 D}$, where $\gamma_{0}\left(\frac{\lambda_{0}}{2 \pi d}\right)^{2}$ can be viewed as the (effective) coupling strength between exciton and photons.

For usual semiconductors, the enhanced factor in Eq. (19) is about $10^{3}$ for Wannier excitons in the optical range. However, due to the extreme smallness of $\gamma_{\text {sin gle }}$ itself, observation of $\Omega_{k_{z} \sim 0, n}^{r e n}$ in a single quantum wire is not expected to be easy. If the decay rate of the exciton is in the order of $\mathrm{ps}^{-1}$, the radiative shift is about $10^{-1} \mathrm{meV}$. To observe the coherent effect, one may increase the number $\left(N^{\prime}\right)$ of the wires. If $N^{\prime}$ parallel quantum wires (with $L_{c}>>\lambda$ ) are placed on the same plane, 
the state of the systems is written

$$
|\psi(t)\rangle=\sum_{j=1}^{N^{\prime}} g_{j}(t)\left|j, k_{z}, n ; 0\right\rangle+\sum_{\mathbf{q}^{\prime}} g_{G ; \mathbf{q}^{\prime} k_{z}}(t)\left|G ; \mathbf{q}^{\prime} k_{z}\right\rangle
$$

where $\left|j, k_{z}, n ; 0\right\rangle$ is the state in which the $j$ th wire is excited with no photon present, and $\left|G ; \mathbf{q}^{\prime} k_{z}\right\rangle$ represents the state in which all wires are unexcited with one photon present. When the wires are distributed in equal spacing as in a lattice, the solution can be written as 19

$$
g_{j}(t)=N^{\prime-1} \sum_{p} e^{i p x_{j}} e^{i \varpi(p) t}
$$

where $x_{j}$ is the position of the $j$ th wire, and the $p$ vector is quantized as usual, obeying the periodic boundary condition. The complex frequency $\varpi(p)$ can be obtained as

$$
\varpi(p)=\sum_{\mathbf{q}^{\prime}} \sum_{j=1}^{N^{\prime}}\left|D_{\mathbf{q}^{\prime} k_{z} n}\right|^{2} \zeta\left(\omega_{\mathbf{q}^{\prime} k_{z} n}\right) \exp \left[i\left(q_{x}^{\prime}-p\right) x_{j}\right],
$$

where the vector $\mathbf{q}^{\prime}=\left(q_{x}^{\prime}, q_{y}^{\prime}\right)$ and the function $\zeta\left(\omega_{\mathbf{q}^{\prime} k_{z} n}\right)=\mathcal{P} / \omega_{\mathbf{q}^{\prime} k_{z} n}-i \pi \delta\left(\omega_{\mathbf{q}^{\prime} k_{z} n}\right)$. One can note that from Eq. (23), as $x_{j} \rightarrow 0$, the frequency shift $\Omega_{k_{z} n}^{\prime} \equiv$ $\operatorname{Re} \varpi(p)=N^{\prime} \Omega_{k_{z} n}$. It means if there are $N^{\prime}$ parallel quantum wires within the cooperative length $\lambda\left(k_{0} x_{j}=\right.$ $\left.\frac{2 \pi}{\lambda} x_{j}<<1\right)$, the frequency shift will be enhanced approximately by an extra factor of $N^{\prime}$ - the number of the wires. Thus, the total enhanced factor to the frequency shift is about $N^{\prime} /\left(k_{0} d\right)=N^{\prime} \lambda /(2 \pi d)$.

In summary, we have studied the radiative decay of the Wannier exciton in a one-dimensional system. It is shown that the radiative frequency shift can be explicitly calculated after a proper renormalization has been made. Similar to its decay-rate counterpart, the renormalized frequency shift is superradiatively enhanced by the factor of $\left(\frac{1}{k_{0} d}\right)$.

One of authors (D. S. Chuu) would like to thank to Prof. Y. C. Lee of SUNYAB for helpfull discussions. This work is supported partially by the National Science Council, Taiwan under the grant number NSC 91-2120M-009-002.
[1] R. H. Dicke, Phys. Rev. 93, 99 (1954).

[2] N. Skiribanowitz, I. P. Herman, J. C. MacGillivrary, and M. S. Feld, Phys. Rev. Lett. 30, 309 (1973).

[3] V. Ernst and P. Stehle, Phys. Rev. 176, 1456 (1968);F. Arechi and D. Kin, Opt. Commun. 2, 324 (1970).

[4] J. J. Hopfield, Phys. Rev. 112, 1555 (1958).

[5] V. M. Agranovich and O. A. Dubovskii, JETP Lett. 3, 223 (1966).

[6] Ya. Aaviksoo, Ya. Lippmaa, and T. Reinot, Optics and Spectroscopy (USSR) 62, 419 (1987).

[7] B. Deveaud, F. Clerot, N. Roy, K. Satzke, B. Sermage, D. S. Katzer, Phys. Rev. Lett 67, 2355 (1991).

[8] J. Knoester, Phys. Rev. Lett. 68, 654 (1992).

[9] G. Björk , S. Pau, J. M. Jacobson, H. Cao, and Y. Yamamoto, Phys. Rev. B 52, 17310(1995).

[10] D. S. Citrin, Phys. Rev.B 47, 3832 (1993).

[11] V. M. Agranovich, D. M. Basko, and O. A. Dubovsky, J. Chem. Phys. 106, 3896 (1997).
[12] A. Nakamura, H. Yamada, and T. Tookizaki, Phys. Rev. B 40, 8585 (1989).

[13] E. Hanamura, Phys. Rev. B 38, 1228 (1988).

[14] F. C. Spano, J. R. Kuklinski, and S. Mukamel, Phys. Rev. Lett. 65, 211 (1990).

[15] Y. C. Lee, D. S. Chuu, and W. N. Mei, Phys. Rev. Lett. 69, 1081 (1992).

[16] D. S. Citrin, Phys. Rev. Lett. 69, 3393 (1992); D. S. Citrin, Phys. Rev. B 48, 2535 (1993).

[17] Y. N. Chen and D. S. Chuu, Phys. Rev. B 61, 10815 (2000); Europhys. Lett. 54, 366 (2001).

[18] Y. C. Lee and D. L. Lin, Phys. Rev. A 6, 388 (1972).

[19] P. S. Lee and Y. C. Lee, Phys. Rev. A 8, 1727 (1973).

[20] V.V. Popova, T.V. Teperika, N.J.M. Horingb, and T.Yu. Bagaeva, Solid State Commun. 127, 589 (2003).

[21] One should note that $\mathrm{Z}$ mode emission drops to zero at normal direction $\left(k_{\|}=0\right)$. 